TRABAJO EN PROGRESO

\title{
Utilización de conceptos de gamificación para reducir la violencia de género contra las mujeres
}

\author{
Cuauhtémoc Rivera-Loaiza, Marco A. Nava Aguilar
}

Publicado: 31 Octubre 2018

\begin{abstract}
Resumen
Uno de los problemas que más preocupan a la Sociedad Mexicana es la violencia de género contra las mujeres, el cual parece ser un fenómeno endémico que ha incrementado su potencial de lastimar personas, con la amplia disponibilidad de las redes sociales. Por tal motivo, se han instituido una gran cantidad de iniciativas para contrarrestar este problema, pero los resultados no han sido los esperados. Nuestra propuesta consiste en concientizar a las generaciones más jóvenes sobre las consecuencias de participar en eventos de violencia de género mediante la utilización de un videojuego que involucra la toma de decisiones conductuales.
\end{abstract}

Palabras clave: Videojuegos, gamificación, violencia de género, diseño centrado en el usuario, interfaces de usuario.

\section{Introducción}

En México, como en la mayoría de los países, la Violencia de Género contra las mujeres (VGCM) es uno de los problemas sociales más extendidos. Es un fenómeno que históricamente ha estado presente y que trasciende cualquier tipo de clase social, cultural y económica. En tiempos recientes diversos casos de VGCM han expuesto la gravedad de este asunto, y se han convertido en temas de discusión en los medios de comunicación tradicionales y, de manera muy particular, en las redes sociales. Durante el último lustro casos como el de Mara Castillo [3] han trascendido el campo criminal para convertirse en movimientos sociales que se han convertido en tendencia en las redes digitales y han puesto, nuevamente, el tema en el discurso nacional.

Sin embargo, esto no ha disminuido la cantidad de mujeres agredidas. Las agresiones a las mujeres se encuentran en mayor medida en ambientes como el escolar, familiar, laboral y comunitario [2]. En los últimos 10 años, contando hasta el 2016, INEGI contabilizó 22 mil 482 mujeres asesinadas en las 32 entidades del país. Comparado a mil 83 mujeres asesinadas en

\footnotetext{
Rivera-Loaiza, C.

Facultad de Ciencias Físico-Matemáticas/ CENIEM

UMSNH

Morelia, México.

Email: crivera@ fismat.umich.mx

Nava Aguilar, MA.

Facultad de Ciencias Físico-Matemáticas

UMSNH

Morelia, México.

Email: marconavaa@hotmail.com
}

2007 a dos mil 735 en el 2016, se tiene la poco agradable realidad de un aumento del $152 \%$. En este recuento de agresiones durante una década en el país, también se da a entender el hecho de que cada 4 horas una niña, joven o mujer adulta fue asesinada en alguna entidad mexicana [9].

De acuerdo con [7] en 2011, 63 de cada 100 mujeres de 15 años y más declaró haber padecido algún incidente de violencia, ya sea por parte de su pareja o de cualquier otra u otras personas. En la figura 1 se presentan las prevalencias totales de violencia contra las mujeres de 15 años y más por tipo de violencia y tipo de agresor ejercidas a lo largo de su vida.

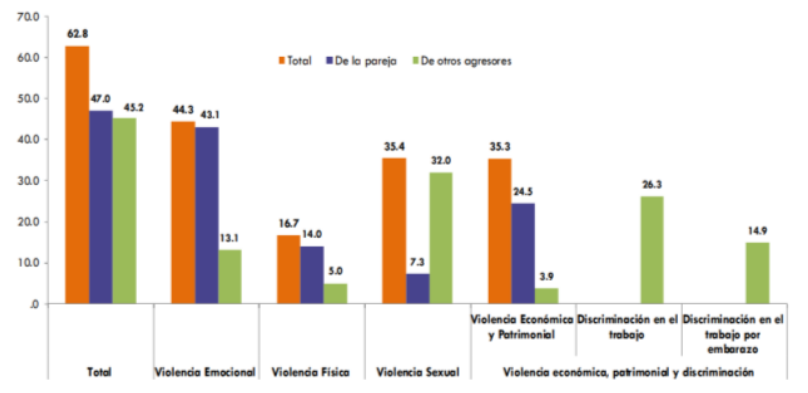

Figura 1. Prevalencias totales de violencia contra las mujeres de 15 años y más por tipo de violencia y tipo de agresor ejercidas a lo largo de su vida (2011) [7].

Durante nuestro proceso investigativo, el cual es documentado en detalle en [11] fue notable la poca efectividad de las políticas establecidas para disminuir la incidencia de la VGCM. Sin embargo, un área en la que existe esperanza en que se logre un cambio a mediano y largo plazo: la atención y concientización sobre el fenómeno en las generaciones más jóvenes.

En este sentido, nuestra propuesta se centra en desarrollar un videojuego dirigido a jóvenes menores de 15 años. El objetivo principal de este juego es la utilización de las estrategias tradicionales de videojuegos para procurar la implantación los principios básicos para evitar la VGCM en los usuarios que utilicen el sistema y potencialmente provocar cambios en la conducta de todos los actores.

\section{Metodología}

Para la elaboración del videojuego decidimos emplear el proceso de desarrollo denominado Diseño Centrado en el Usuario. Conocido mejor por sus siglas en inglés, User Centered Design (UCD) es una metodología que describe los procesos de diseño 
que los usuarios finales influyen en cómo un diseño toma forma [1]. Es una filosofía de diseño que tiene por objetivo la creación de productos que resuelvan necesidades concretas de sus usuarios. Además, hacemos uso de principios básicos de gamificación para procurar una efectividad de nuestra estrategia con preadolescentes.

En el caso de UCD, esta trata de desarrollar productos que se basan en la información acerca de las personas que finalmente utilizarán el producto. Esta forma de desarrollo de productos se basa en teorías desarrolladas en la interacción humanocomputadora, el diseño industrial y la psicología cognitiva. UCD tiene fundamentalmente cuatro etapas [15]: Especificación del contexto de uso: identificar quienes son los principales usuarios del producto, para qué lo van a utilizar y en qué condiciones lo utilizarán. En base a lo anterior, procedimos a identificar plenamente el contexto en que se utilizaría el sistema y establecer el estatus quo del problema de la violencia de género en el estado de Michoacán.

En este proyecto el tema de gamificación fue primordial también. Gamificación es una propuesta de diseño que involucra la utilización de elementos de videojuegos (games, en inglés; de ahí el nombre de gamification) en contextos no necesariamente con fines lúdicos [5][6]. Creemos que al involucrar elementos de videojuegos en contextos que no tienen por intención únicamente el ocio, sino el procurar -en nuestro caso, por ejemplo- el cambio de conductas mediante la recompensa por la elección de buenas prácticas conductuales es una estrategia que puede tener consecuencias muy positivas. Esto aunado a la familiaridad que los usuarios del sistema tienen con los videojuegos, la curva de aprendizaje de nuestro videojuego sería muy breve. En el caso de nuestro proyecto, los usuarios obtendrán puntos dependiendo de su actuar en la historia del videojuego.

\section{Entrevistas}

Dentro de esta primera etapa en nuestro proceso de investigación se procedió a realizar una investigación exhaustiva sobre el tema, centrándonos en la situación de la violencia de género en Michoacán. Por las características de este problema en nuestro país, la investigación no sólo se limitó a la consulta estadística, sino que además se investigaron diversas fuentes periodísticas relativas a casos de violencia de género hacia las mujeres que se han presentado en los últimos meses. Se realizaron cinco entrevistas: tres con funcionarias estatales y dos con estudiantes de la Universidad Michoacana. Las dos estudiantes fueron explícitamente seleccionadas por haber enfrentado situaciones de violencia de género dentro de la propia Universidad.

En el caso de las entrevistas con las funcionarias fue notoria la discordancia entre las leyes (o protocolos, en el caso de la Universidad Michoacana) escritas y la práctica. Las funcionarias tenían cargos directivos en la Universidad Michoacana, en la Casa de la Cultura Jurídica y el Centro de Justicia Integral para las Mujeres, todas estas instancias pertenecientes al sector público; en todos los casos sus cargos tenían injerencia con la aplicación y diseño de políticas para la atención del problema de la violencia de género. En principio, un punto común entre ellas es que independientemente de su situación laboral o profesional, las funcionarias sufrían de acoso continuo.

Las alumnas entrevistadas han sufrido de situaciones de violencia de género dentro de la Universidad. En ambos casos se trató de una situación de acoso por parte de un docente, y procedieron a presentar una denuncia. Pese a contar con un Protocolo de Atención a Víctimas de Violencia de Género, en la práctica este es inoperante.
Las entrevistas nos permitieron llegar a la conclusión de que la única forma en que puede lograrse un cambio es concientizando a las nuevas generaciones. Las personas que pertenecen a otros sectores de la población difícilmente van a cambiar sus actitudes, pese a las posibles sanciones legales que les pudieran aplicar.

\section{Pruebas de usabilidad con prototipos de baja fidelidad}

Los prototipos de baja fidelidad son ampliamente utilizados para el desarrollo de sistemas informatizados. Un prototipo de baja fidelidad es aquel que tiene generalmente funciones limitadas de interacción y son primordialmente usados para probar conceptos, alternativas de diseño y secuencias de interacción [12]. Una de las variantes de las técnicas de prototipado de baja fidelidad es la construcción de prototipos en papel, los cuales ayudan a los diseñadores y desarrolladores a ver de qué manera los usuarios usan y reaccionan a una interfaz sin necesidad de construir el sistema en su totalidad [10].

Los prototipos en papel son una técnica que involucra solamente dibujos sobre papel, generalmente a mano alzada. Esto permite que la prueba de usabilidad se centre en las funcionalidades del sistema y no en la forma en cómo finalmente se verá el producto final [8].

Para esta etapa se reclutaron a 5 usuarios con edades entre los 12 y 15 años. De estos dos eran hombres y tres mujeres. Todos ellos contaban con un teléfono inteligente y experiencia previa en el uso de videojuegos. La duración de cada prueba fue de aproximadamente 20 minutos y fue realizada en las instalaciones de una escuela secundaria en la ciudad de Morelia en enero de 2018. (Figura 2).
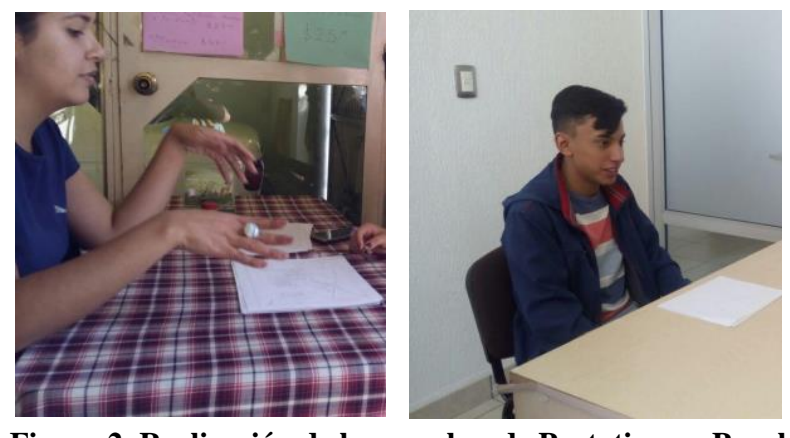

Figura 2. Realización de las pruebas de Prototipo en Papel

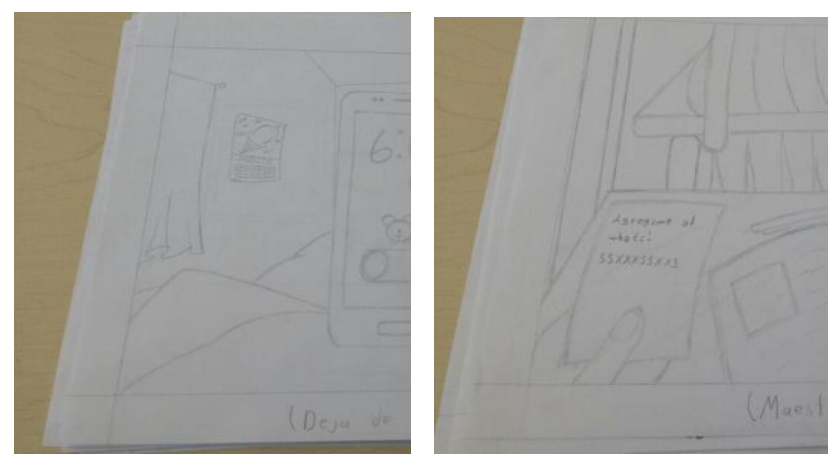

Figura 3. Pantallas del prototipo en papel representando dos escenas del videojuego.

El prototipo en papel estaba compuesto por 16 pantallas, dibujadas a mano, que mostraban la secuencia del juego. El videojuego estaba diseñado desde la perspectiva del jugador en Primera Persona y la primera escena correspondía al momento en 
que el jugador despertaba, y las siguientes escenas donde el usuario debía de tomar decisiones frente a situaciones de violencia de género (Figura 3).

Dependiendo de la decisión tomada, esta era analizada para determinar si, de acuerdo con recomendaciones avaladas por expertos en el tema de violencia de género, su respuesta había sido apropiada y de no serlo, se le mostraban alternativas para cambiar su comportamiento dentro del juego; cabe hacer mención que cuando el comportamiento no era el correcto la consecuencia de su decisión era mostrada y posteriormente se le daba la oportunidad de cambiar su decisión por una acción adecuada.

\section{Diseño de prototipos de alta fidelidad}

La siguiente etapa del proceso de desarrollo consiste en la creación de prototipos de alta fidelidad, mediante una herramienta de software que nos permite emular la experiencia del videojuego en los dispositivos para los que sería diseñado, que en este caso serían teléfonos inteligentes. Para esto elegimos hacer uso de POP de la compañía Marvel (https://marvelapp.com/pop/), que nos permite hacer una actividad muy similar al prototipado en papel, pero incorporando elementos interactivos que son utilizados dentro del dispositivo mismo. Esto permite una mejor experiencia de usuario, pues se acerca de una manera mucho más fiel a lo que sería el sistema en su etapa final. Las Figuras 4 y 5 muestran algunas de las pantallas utilizadas en esta etapa de prueba.

Las pruebas de usuarios con esta herramienta se encuentran en proceso de implementación durante el año escolar que inicia en el mes de agosto de 2018. El prototipo de alta fidelidad se muestra, en uso, en la Figura 6. De manera similar al prototipado en papel, se harán pruebas con al menos cinco estudiantes de secundaria, localizados en la ciudad de Morelia.



Figura 4. Escena 1 del videojuego, centro comercial.

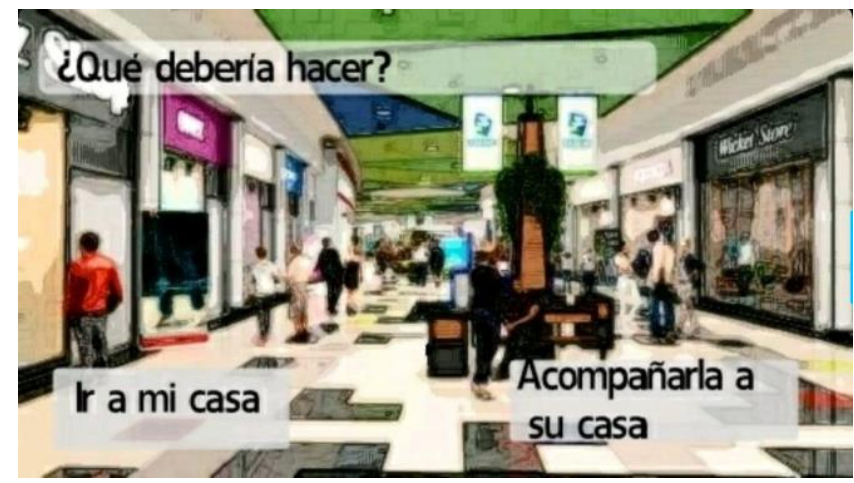

Figura 5. Escena 2 del videojuego, centro comercial.
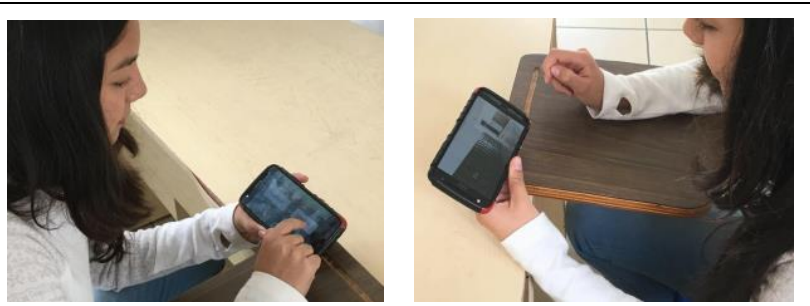

Figura 6. Prototipo de alta fidelidad.

\section{Conclusiones y trabajo futuro}

Las pruebas de usabilidad realizadas con los prototipos de baja fidelidad nos dieron información sumamente valiosa, la cual reafirma nuestra estrategia de diseño. Los usuarios mostraron gran interés en la plataforma, la cual era muy familiar (en particular para los varones) e incluso sugirieron varios escenarios que no teníamos contemplados inicialmente y que pensamos incorporar en la versión final. En entrevistas posteriores al uso de la herramienta, los usuarios manifestaron un nivel de concientización sobre sus acciones y las consecuencias que estas pueden tener sobre otras personas.

Posterior a la culminación de la etapa de pruebas de prototipo de alta fidelidad procederemos a la programación del videojuego. Para esto se tiene contemplado trabajar inicialmente sobre la plataforma Android, y contar con el producto final en el último trimestre de 2018.

\section{Referencias}

[1] C. Abras, D. Maloney-Krichmar y J. Preece. (2004). "Diseño centrado en el usuario"

http://citeseerx.ist.psu.edu/viewdoc/download?doi=10.1.1.94. $381 \&$ rep=rep1\&type=pdf (Consultado el 27 de febrero de 2017)

[2] K. Blondeel et al. "Violence motivated by perception of sexual orientation and gender identity: a systematic review". Bulletin Of The World Health Organization . January 2018;96(1):29-41E. Available from: Academic Search Complete, Ipswich, MA. Consultado el 4 de abril, 2018.

[3] CNN. 2017. 5 Claves del feminicidio de Mara Castilla, el crimen que sacude a México.

https://cnnespanol.cnn.com/2017/09/18/cinco-claves-delfeminicidio-de-mara-castilla-el-crimen-que-sacude-amexico/ Obtenido en agosto de 2018.

[4] Delamere, F. M. 2004. "It's just really Fun to Play"! A Constructionist Perspective on Violence and Gender Representations in Violent Video Games. Order No. NQ94570, University of Waterloo (Canada), Ann Arbor, 2004.

[5] Deterding, S., D. Dixon, R. Khaled, and L. Nacke. 2011. From game design elements to gamefulness: defining "gamification". In Proceedings of the 15th International Academic MindTrek Conference: Envisioning Future Media Environments (MindTrek '11). ACM, New York, NY, USA, 9-15. DOI: https://doi.org/10.1145/2181037.2181040

[6] Ejsing-Duun, Stine \& Skovbjerg, Helle Marie. (2014). Gamification of a higher education course: What's the fun in that?. Proceedings of the European Conference on Gamesbased Learning. 1. 92-98.

[7] INEGI. "Estadísticas A Propósito Del Día Internacional De La Eliminación De La Violencia Contra La Mujer (25 De 
Noviembre)". Instituto Nacional de Estadística y Geografía. http://www.inegi.org.mx/saladeprensa/aproposito/2016/viole ncia2016_0.pdf (3)

[8] Y. Li, Xiang Cao, Katherine Everitt, Morgan Dixon, and James A. Landay.. "FrameWire: a tool for automatically extracting interaction logic from paper prototyping tests". In Proceedings of the SIGCHI Conference on Human Factors in Computing Systems (CHI '10). ACM, New York, NY, USA, 2010, 503-512. DOI:

https://doi.org/10.1145/1753326.1753401 (4)

[9] M. Muedano. "Imparable, el crimen contra las mujeres; cifras del Inegi". Excelsior. México, 2017.

http://www.excelsior.com.mx/nacional/2017/10/22/1196308 Consultado el 15 de febrero de 2018. (5)

[10] W. Preston, James Goulding, Gary Burnett, and Mike Jackson. "Heuristics \& usability of virtual attack points for pedestrian navigation: user study using paper-prototyping". In Proceedings of the First ACM SIGSPATIAL International Workshop on Mobile Geographic Information Systems (MobiGIS '12). 2012. ACM, New York, NY, USA, 65-72. DOI=http://dx.doi.org/10.1145/2442810.2442822 (6)

[11] Rivera-Loaiza, C. et al. 2018. Diseño de un videojuego para reducir la violencia de género entre los infantes. XV Encuentro Participación de la mujer en la ciencia. Avances de la Ciencia en México. ISBN: 978-607-95228-8-9

[12] J. Rudd, Ken Stern, and Scott Isensee. "Low vs. high-fidelity prototyping debate". interactions 3, 1 (January 1996), 76-85. DOI=http://dx.doi.org/10.1145/223500.223514 (7)

[13] Usability.gov "User-Centered Design Basics", https://www.usability.gov/what-and-why/user-centereddesign.html Obtenido en Mayo de 2017. (9) 